Introduction: Providing families with a safety net of information is a crucial component in the care of children with acute illnesses at home (Jones et al 2013). Safety netting is recommended by UK national bodies and has been found to reduce re-consultation safely (Maguire et al 2011).

Aim: To provide parents with information to help them determine when to seek help for an acutely ill child.

Methods: Research programme encompassed five projects: a systematic review; a qualitative exploration of safety netting information used by parents and professionals; a mixed methods development of content, format and delivery modes for the first intervention; video capture of children with acute illness; and co-design of the content of the intervention with parents and professionals.

Conclusion: ASK SNIFF projects repeatedly demonstrate the need for professionally endorsed, co-produced safety-netting resources for parents with varying information needs, literacy levels and ability to use information technology.

\title{
OC26 - Parents of young children in research: informants, consultants and collaborators
}

\author{
Sarah Neill (United Kingdom) ${ }^{1}$; Damian Roland (United Kingdom)2; \\ Matthew Thompson (United States of America); ${ }^{3}$ Natasha Bayes \\ (United Kingdom) $)^{1}$; Laura Mullins (United Kingdom) ${ }^{4}$; \\ Monica Lakhanpaul (United Kingdom) ${ }^{5}$
}

${ }^{1}$ University of Northampton; ${ }^{2}$ University of Leicester and University Hospitals Leicester; ${ }^{3}$ University of Washington; ${ }^{4}$ Lay member; ${ }^{5}$ University College London

Theme: Ethical issues: dignity and humanity.

Keywords: Parents, research participation; research involvement; research collaboration.

Introduction: Patient and public involvement in research is recognised as best practice in the UK, as services developed with service users are more likely to meet their needs. In child health this often means engaging with parents of young children.

Aim: To share experiences of engaging with parents of young children in research projects at varying levels of participation.

Methods: Methods include parents as participants, as parent panels and as research team members reflecting various steps on the participation ladder (Hart 1992).

Results: Parents report personal benefits and a range of different challenges when engaging in child health research which impact the management/delivery of research projects.

Conclusion: Key elements of working with parents include time to plan involvement, building respect/mutual understanding, equal access to project activities, continual support for parents, reward and recognition. Research projects need to cost and plan for parents' engagement in research for it to be successful. 